\section{Diferencia y Educación: Implicaciones del reconocimiento del otro}

Difference and education: Implications of recognition of the other

\author{
Diferença e educação: \\ implicações do \\ reconhecimento do outro
}

\section{Sandra Guido Guevara*}

* Doctora en Educación. Profesora Departamento de Posgrados, Facultad de Educación. Grupo de investigación: Equidad y diversidad en Educación. Universidad Pedagógica Nacional. Correo electrónico: sguido@pedagogica.edu.co

\section{Resumen}

Este artículo presenta el contexto político y educativo donde se cuestiona la educación homogénea y se introduce lo diverso. Posteriormente, desarrolla tres elementos que limitan la construcción de espacios pedagógicos con la diferencia: el origen de la escuela, la prevalencia del discurso de la diversidad sobre el de la diferencia y el desconocimiento de aspectos sensibles en la relación con el otro. Finalmente, se argumenta a favor de cambios profundos en estos tres aspectos y de cómo el trabajo educativo con la diferencia enriquece la práctica pedagógica.

\section{Palabras Claves}

Educación y diferencia, educación y diversidad, pedagogía y alteridad.

\section{Abstract}

This article presents the political and educational context where homogeneous education is asked and the diverse is introduced. Consequently, it develops three elements that prevent from the construction of pedagogical spaces with the difference: origin of the school, prevailing of the speech of diversity over the one of the difference, and ignorance of sensitive aspects about the relation to the other. Finally, it is argued for the deep changes in these three aspects and how educational work with the difference enriches the pedagogical practice.

\section{Key Words}

Education and difference, education and diversity, Pedagogy and alternation.

\section{Resumo}

Neste artigo apresenta-se o contexto educacional e político no qual se questiona a educação homogênea e se introduze a diversidade. Posteriormente, se explicam três elementos que limitam a construção espaços de ensino na diferença: a origem da escola, a prevalência do discurso da diversidade sobre o discurso da diferença e o desconhecimento de aspectos sensíveis na relação com o outro. Finalmente, se defende a introdução de profundas mudanças nestes aspectos e a idéia de que o trabalho educativo com a diferença enriquece a prática pedagógica.

\section{Palavras-chave}

Educação e diferença, educação e diversidade, pedagogia e alteridade.

Fecha de recepción: 30 de julio de 2010 Fecha de aprobación: 18 de octubre de 2010 
1. El origen de la escuela y su propósito que ha tenido desde su raíz una dificultad para trabajar con el otro, con el "diferente".

2. La idea que revelan políticas sociales, y en general campañas internacionales, relacionada con el valor de la diversidad; esto ha implicado actualizar el discurso de la diversidad y no el de la diferencia.

3. El desconocimiento de lo imbricado en el interior del ser de aquello que nos hace sentir el otro: su olor, su color, su cuerpo, su idioma, su cosmovisión, su ritmo y forma de aprender, su distancia; es decir, su diferencia; además de la representación que se tiene del otro relacionada con los sistemas de significación construidos en la interacción social.

Estos impedimentos se presentarán en lo que sigue como elementos de reflexión y, luego, de posible acción para una experiencia educativa más responsable con el otro.

\section{Romper con los principios desde los cuales se creó la escuela}

La educación es el punto en el que decidimos si amamos el mundo lo bastante como para asumir una responsabilidad por él y así salvarlo de la ruina que, de no ser por la renovación, de no ser por la llegada de los nuevos, sería inevitable.

\section{Hanna Arendt}

Hablar de educar al otro en la escuela se convierte en un reto que rompe con sus orígenes ${ }^{3}$. La escuela, como institución, se reconoce como una de las mayores reivindicaciones de la modernidad al crearse como la posibilidad de integrar al conjunto de los sujetos a un proyecto nacional; la escuela se hizo necesaria para configurar el Estado-Nación occidental (Álvarez, 1995; Pineau, 1996; y Duschatsky, 1999).

La escuela se creó desde la lógica del progreso, la civilización y la evolución; para ello, sus prácticas giran en torno a la instrucción. En este sentido, aquellos otros que en su propio tiempo y modo enrarecen, detienen o lentifican esa mirada prospectiva hacia el progreso, aquellos que rompen por su "raza", por su "comportamiento", por "su forma de estar y aprender" con los cánones de lo moderno -es decir, de un pensamiento eurocéntrico-se constituyen en impedimento para alcanzar el propósito de la escuela misma.

3 Es de aclarar que la educación se refiere a un proceso general de la experiencia humana. Por su parte, la escolarización se constituye en uno de los recortes de este proceso educativo más amplio (Lino, 2000). "...aquellos otros que en su propio tiempo y modo enrarecen, detienen o lentifican esa mirada prospectiva hacia el progreso, aquellos que rompen por su "raza", por su "comportamiento", por "su forma de estar y aprender" con los cánones de lo moderno -es decir, de un pensamiento eurocéntrico- se constituyen en impedimento para alcanzar el propósito de la escuela misma."

En este sentido, algo se tiene que hacer con la diferencia: invisibilizarla, excluirla, eliminarla. De acuerdo con Castro-Gómez (2005), la construcción del perfil de subjetividad que requería el proyecto moderno exigía la supresión de todas las diferencias.

La escuela, en búsqueda de su ideal, ha priorizado una lengua -la hispana-, una forma de pensamiento racional -heredado de la Ilustración- y un sujeto -moderno: hombre, blanco, cristiano, heterosexual, letrado-. La escuela se ha mostrado como un dispositivo útil para normalizar, "esto es, homogenizar. Pero también implícitamente, está diferenciando entre civilizados e incivilizados, viciosos y virtuosos, ignorantes y letrados" (Álvarez, 1995, p. 92). Así, desconoce otros lenguajes, otras formas de pensar, otras maneras de ser y de sentir. Decir ahora que se respeten la lengua, los ritmos y las formas de aprender, el pensamiento propio, los otros cuerpos con sus maneras de percibir, de andar, de estar, implica empezar a derribar la columna vertebral sobre la cual ha caminado la escuela desde su creación.

Se podría decir que, luego de que la escuela inhabilitó el diálogo con lo plural, pretender eliminar lo negativo, normalizar lo anormal, desconocer lo diferente y educar para la constitución de "sujetos civilizados" mediante una educación homogénea y masiva que reclama su reconocimiento en medio de las condiciones actuales de la educación, o por lo menos de la educación pública: aulas con más de 40 estudiantes, currículos y programas que no responden a las realidades sociales, culturales y económicas de los estudiantes y, por lo tanto, distancias cada vez más amplias entre la cultura escolar y la de sus propias vidas -además, de tiempos inflexibles y criterios de rendimiento también descontextualizados que, de por sí, ya inhabilitan este propósito-. 
Se ha de aclarar que, desde su creación, la escuela ha atravesado por momentos distintos entorno al trabajo con la diferencia. Los movimientos sociales y las comunidades organizadas han visto en el proceso educativo uno de los fundamentos de su resistencia que, aun cuando no sea el objeto de este escrito, se hace pertinente destacar que sus propuestas educativas y la investigación de las mismas son fundamentales para develar la otra cara de la moneda.

\section{Hablar de diversidad; no de diferencia}

¿Cuántos soy? ¿Quién es yo? ¿Qué es ese intervalo que hay entre mí y mi?

Fernando Pessoa

Existe una gran disimilitud entre hablar de diversidad y de diferencia. La diversidad se relaciona con una condición, con una realidad que reconoce la pluralidad desde un lugar particular, desde el lugar de la hegemonía cultural. Frente a esta diversidad, cuando aparecen los otros, se hace necesario integrarlos, asimilarlos y normalizarlos, unificarlos a una totalidad de pensamiento. Lo que Occidente ha hecho con el otro, según Skliar (2206) no es más que una intención por reducir todo otro radical en otro próximo"4. Y transformar la alteridad radical en próxima es una tentación difícil de evitar, pues el otro, radicalmente diferente, representa siempre una perturbación a nuestra identidad, una amenaza a la construcción "armónica" de imagen de grupo, comunidad, nación, etc. Sin embargo, a pesar del propósito de asimilar y unificar, existen cosas del otro que son siempre irreductibles.

Con el fin de aproximar al otro en el campo educativo, se le caracteriza, estudia y clasifica; en otras palabras, se le tematiza. En este sentido, se crean estrategias de formación donde se estudia al otro en su rareza; así se configura, según Skliar (2006), un discurso técnico que banaliza y clasifica la diferencia.

La diversidad y los sujetos que la integran se definen por un nosotros que se ubica en el lugar de

4 El resaltado en cursivas corresponde a la autora. la supremacía cultural: existen unos lugares y unos sujetos que deciden lo bueno y lo malo, lo normal o lo anormal: "[...] la modernidad inventó y se sirvió de una lógica binaria a partir de la cual denominó de distintos modos al componente negativo de la relación cultural: marginal, indigente, loco, deficiente, drogadicto, homosexual, extranjero, etc." (Duschatzky y Skliar, 2001, p. 191). La diversidad se atribuye a sujetos y a grupos; en este sentido, se ratifica un nosotros y un otros. Los diversos son los indígenas, las personas con "necesidades educativas especiales", los campesinos, los afrocolombianos, etc.; y estos grupos o personas son objeto de normalización y responsables de acomodarse a lo existente como hegemónico ${ }^{5}$.

El nosotros no tiene que movilizarse ni, mucho menos, transformarse; escasamente hará algo para acercar al diferente al nosotros. Y el otro es factible de acercar, pero no de cualquier forma, no a cualquier precio. La diversidad y el accidente son posibles de incorporar, siempre y cuando no se rompa el orden interno de la experiencia y sus jararquizaciones

5 A continuación, Según Guido y otros (2010) se ofrece un ejemplo de cómo en la política educativa colombiana se evidencia la atribución de la diferencia a los diferentes y no a todos los miembros de una sociedad:

El concepto de interculturalidad ha estado ligado históricamente a la educación para grupos étnicos y a proyectos de etnoeducación como es afirmado por Castillo y Caicedo (2008). En el campo de la etnoeducación la idea de la interculturalidad se configura como la capacidad de los sujetos étnicos para interactuar con otras culturas y como cualidad de los sujetos y saberes.

Así lo reflejan las políticas: de acuerdo con los lineamientos de política para poblaciones vulnerables (2005) “la prestación del servicio etno-educativo se debe fundamentar en los principios de interculturalidad" (pp 17-18). En la Cátedra de estudios afro colombianos, la interculturalidad es catalogada como un principio de la etnoeducación, en correspondencia con el decreto 84 de 1995. De igual forma, en la normatividad básica para etnoeducación se define la interculturalidad como uno de sus principios. Los anteriores elementos dan cuenta del hecho de atribuir la interculturalidad a un factor específico de la educación para grupos étnicos, y no como a una preocupación y puesta en marcha de proyectos pedagógicos para "todos". En este caso la interculturalidad favorece a los grupos étnicos pero deja intactos a los demás. 
de valor asociadas. En "ello fundan su poder las técnicas de disciplinamiento y los límites de todo orden social para absorber y normalizar las anormalidades" (León, 2009, p. 74). La educación, en este sentido, tiene la responsabilidad de construir el camino y habilitarlo para que los otros hagan parte del nosotros. De esta manera se alcanzaría la tan anhelada formación ciudadana y el progreso de las "naciones".

En las políticas educativas colombianas se hace evidente este propósito: la educación debe restablecer, apaciguar y unificar las diferencias a través del diálogo, la tolerancia y la inclusión. Se debe evitar el conflicto y promover la armonía; al respecto se ha de recordar que de acuerdo con Skliar (2008) una relación de alteridad sin conflicto no es una relación de alteridad: es una relación consigo mismo.

Dentro de las políticas de la diversidad, el otro es cambiante, se fabrica cada día otro diferente. Así, en la política educativa colombiana actual, la categoría de poblaciones vulnerables se encuentra compuesta por grupos de gran variabilidad y se le van adicionando cada vez más. -Nótese la inclusión en la política distrital del grupo LGBT, o cómo desde un momento coyuntural en lo político y social en Colombia empiezan a aparecer como vulnerables los afectados por la violencia-. De otra parte, el reconocimiento específico a las culturas juveniles es declarado explícitamente como grupo especial de atención en la política de Bogotá, por ser una preocupación del mundo urbano. De igual forma, es interesante ver cómo las denominaciones se van transformando de acuerdo con los cambios que circulan en las comunidades académicas -por ejemplo, el concepto de personas con limitaciones luego se cambia por el de necesidades educativas especiales; esto atiende a la transformación de un concepto biologicista de la discapacidad por el de uno más holístico y social- ${ }^{6}$.

Esto sucede por lo que Skliar (2006) ha denominado obsesión por los extraños -cuando nos obsesionamos por algo, aparece cada vez más-. De acuerdo con este autor, la escuela no se ha preocupado por la diferencia sino por el diferencialismo. Así, los otros se ubican en una condición peyorativa, negativa y subalterna que atiende a una condición binaria: bueno-malo, normal-anormal, negativopositivo, correcto-incorrecto. De esta forma, cada vez se encuentran más grupos y condiciones que aumentan el número de los otros y que pueden justificar la razón de ser de muchas instituciones, profesionales y proyectos.
La diferencia, por el contrario, habla de una distancia con el otro que se reconoce y se interioriza al establecer vínculos con experiencias de vida que conversan con las alteridades. La diferencia no se ubica en algunos sino que es una responsabilidad de todos y no da lugar a supremacías culturales. Desde la diferencia se discuten las consecuencias pedagógicas de un pensamiento occidental binario que propende por la identificación y el señalamiento de lo normal-anormal y, según el cual, se privilegia el primer concepto sobre el segundo y se desencadenan relaciones de exclusión, discriminación, negación e incluso violencia hacia el otro.

"La diferencia, por el contrario, habla de una distancia con el otro que se reconoce y se interioriza al establecer vínculos con experiencias de vida que conversan con las alteridades. La diferencia no se ubica en algunos sino que es una responsabilidad de todos y no da lugar a supremacías culturales."

La diferencia destaca el conflicto, la lucha, la negociación. Es decir, consiste más en una relación que una atribución a sujetos y grupos. Es un asunto de todos, no de los diferentes o de los que estudian a los diferentes. Así, situados en una relación con la diferencia que altera, genera conflictos y no constituye una atribución de sujetos y grupos -en una posición que valora al otro y no lo polariza desde una supremacía cultural- puede observarse con una mirada crítica la postura romántica de la diversidad en la cual es suficiente relacionarme con el otro desde la tolerancia y el reconocimiento.

Skliar (2000) propone una serie de características sobresalientes de la diferencia: no se constituye en una obviedad cultural; se construye histórica, social y políticamente; siempre es diferencia, no se la puede entender como algo impropio que debe volver a la normalidad; es política y no atiende solo a diferencias formales, textuales o lingüísticas; no puede verse como totalidad, no se permea de fácil manera ni pierde de vista sus propias fronteras; no depende de una autorización o de un permiso otorgado desde la normalidad sino de su reconocimiento político y práctico. 


\section{Relacionarse con el otro con el desconocimiento de aspectos sensibles}

Podemos neutralizar momentáneamente uno o más de nuestros sentidos pero con ello solo lograremos agudizar los demás.

No hay modo de comprender el mundo sin detectarlo antes con el radar de los sentidos.

\section{Diane Ackerman}

En este apartado se desarrollan dos ideas fundamentales: la primera, las relaciones con el otro, además de estar permeadas por aspectos políticos, sociales y económicos, también tienen que ver con elementos sensibles que deberían ser reconocidos en el marco de posibilitar otras pedagogías. La segunda, el reconocimiento y la relación con el otro se hacen necesarias como una reafirmación de la propia identidad. La alteridad del otro para Larrosa y Pérez, (1998), permanece reabsorbida en nuestra identidad y la refuerza, la hace posible, más arrogante, más segura y más satisfecha de sí misma.

De manera regular, el otro se asocia a la metáfora del extranjero, del extraño y lo extraño. De acuerdo con Sabido (2009), se relaciona con lo lejano, lo desconocido. Lo incierto puede ser exótico y atractivo pero, también, puede llenar de incertidumbre, miedo y repulsión.

A pesar de que lo extraño ha sido asumido como algo propio del otro, la extrañeza no consiste en una característica de sujetos o grupos; se da en medio de una relación con algo que no es familiar, es decir, conocido, cercano y confiable. "No hay extraños en sí, sino extraños para alguien” (Sabido, 2009, p. 31). Si la extrañeza fuera algo propio del sujeto, no se podría explicar cómo esta relación se modifica con el tiempo o se genera de manera distinta en la familia, la escuela, la comunidad, los medios de comunicación, etc. En diversas ocasiones, el extraño empieza a ser menos extraño, incluso querido por los miembros de la comunidad, aunque las relaciones sociales de poder sigan intactas.

Se hace necesario destacar que el encuentro con el extraño se halla inmerso en una relación de poder asimétrica en varios sentidos: políticos, culturales, económicos y sensoriales. Las relaciones con el otro históricamente han devenido de manera desigual, condición que se ha reproducido socialmente: "En el encuentro con alteridades ajenas y extrañas, el criterio de corte estará siempre en función del supuesto de humanidad que un grupo ha decidido que es el que mejor expresa y afirma su existencia y su naturaleza." (León, 2009, p. 69). La educación se ha convertido en uno de los medios principales para la reproducción de esta imagen.

El reconocimiento de los otros permea el orden de lo sensible que muchas veces, tal vez de manera inconsciente, se desconoce o se ignora:

En relaciones particulares en las que el desequilibrio de poder se asocia con algún componente en específico, mirar de cierta manera, juzgar los olores del otro, rechazar su comida, despreciar su música o evitar el mínimo contacto, produce sentido. Es decir; mediante los sentidos corporales y su uso se generan pautas de orientación que emiten la marca 'no acepto cohabitar a tu lado (Sabido, 2009, p. 41).

Si se quiere transformar las relaciones con el otro y proponer opciones educativas distintas, se hace necesario reconocer las condiciones de la relación con el otro: la mirada hacia el otro, la no mirada, es decir, la distancia visual; las reacciones y las sensaciones frente a su olor; el ruido que producen su lengua, su música, sus formas de comunicar; y la repulsión frente a su contacto físico, su cercanía y la posibilidad de tocarlo o de ser tocado por ese otro. De la misma manera, atender el marco históricocultural donde se instalan.

En el orden sensible se encuentra la historia social y la manera como los sentidos corporales han sido constituidos. Para Sabido (2009), los "sentidos del sentido" marcan distancias y proximidades sociales, movilizan relaciones de poder, significan familiaridades y extrañezas, ponen límites para determinar lo propio y lo ajeno, lo uno y lo Otro, lo amado y lo odiado, lo normal y lo anormal, lo superior y lo inferior, lo bueno y lo malo.

Cuando se descifran los sentimientos frente al otro, a su cultura, su lengua, su cuerpo se inicia un acercamiento distinto que identifica cómo interiormente se pueden empezar modificaciones o reafirmaciones en este encuentro.

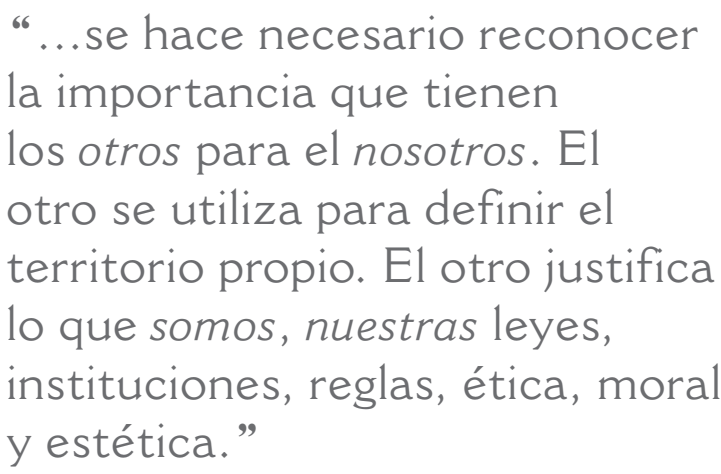
la importancia que tienen los otros para el nosotros. El otro se utiliza para definir el territorio propio. El otro justifica lo que somos, nuestras leyes, y estética." 
Desde otra instancia, se hace necesario reconocer la importancia que tienen los otros para el nosotros. El otro se utiliza para definir el territorio propio. El otro justifica lo que somos, nuestras leyes, instituciones, reglas, ética, moral y estética. Así "El loco confirma nuestra razón; el niño nuestra madurez; el salvaje nuestra civilización; el marginado nuestra integración; el extranjero nuestro país; el deficiente nuestra normalidad" (Duschatzky y Skliar, 2001, p. 192).

El otro se hace necesario porque alguien tiene que portar las desviaciones para justificar los lugares y el trabajo de aquellos que los corrigen, de lugares y personas para hacerlo como el hospital, la cárcel, la escuela, el sanatorio, el maestro, el terapeuta, etc. En este sentido, la escuela se convierte en el camino para transformar lo no deseable, lo anormal, lo extraño en sus contrarias oposiciones.

“[...] los Otros son excepciones que confirman la regla de un mundo necesitado de ordenamiento para habitarlo con sentido. Un mundo donde esos otros carecen de condiciones para existir en el reino de las cosas que deben ser amadas, "podrían no ser nada, porque no son bellos, ni verdaderos, ni reales." (León, 2009, p. 78).

Sin embargo, el otro también se hace necesario para cuestionar mis propias formas, para enriquecer mi horizonte de lo posible.

\section{Cerrar es abrir}

Se trata de que se acepte "al otro" en su extrañeza y en la soberanía de su diferencia.

\section{Edmond Jabés}

El trabajo educativo con el otro no se puede reducir a una experiencia escolar de cercanía con la diferencia. Como se ha venido argumentando, existe una necesidad de movilizaciones profundas en las representaciones, en la sensibilidad, en las bases de la escuela misma que van mucho más allá de lo que las políticas educativas plantean: aceptar al otro, tolerarlo, darle un lugar, es decir, un cupo para que aparezca en el aumento de cobertura educativa, lo cual no significa relacionarme con él.

En su concreción, el trabajo con la diferencia educativa implica preguntarse primero acerca de qué pienso del otro, por lo que me hace sentir, por lo que moviliza en mí en la institución educativa, en la sociedad. Este análisis incluye una mirada crítica del tratamiento que la sociedad, de manera histórica, le ha dado al otro y también una reflexión desde el interior de cada maestro. El trabajo educativo con la diferencia se torna enriquecedor y muy importante para la formación, porque:

- Al entender de manera positiva y real la diferencia se pueden cuestionar nociones y prácticas propias.

- Al trabajar por la ruptura de estereotipos y prejuicios en relación con el otro, se puede evitar la discriminación.

- Al relevar las huellas de los otros en uno mismo, se analizan rasgos comunes.

- Al desarrollar la capacidad crítica, se pueden analizar textos, contenidos escolares y actitudes en relación con el otro.

- Al acercarse al otro, se pueden investigar desequilibrios sociales, trayectorias históricas y resistencias de los movimientos sociales, comunidades, asociaciones, etc.

"...existe una necesidad de movilizaciones profundas en las representaciones, en la sensibilidad, en las bases de la escuela misma que van mucho más allá de lo que las políticas educativas plantean: aceptar al otro, tolerarlo, darle un lugar, es decir, un cupo para que aparezca en el aumento de cobertura educativa, lo cual no significa relacionarme con él."

Asimismo, se hace imprescindible reconocer que para el ser humano existe, de acuerdo con León (2009), una invalidez constitutiva para afrontar -sin sensación caótica y sin temor amenazante con poderes deformantes- variedades extrañas, combinaciones no probadas y menos aún dominios ininteligibles que ofrecen las alteridades humanas. La complejidad del encuentro se hace, entonces, natural y necesaria de descifrar en todas sus aristas, esta es la invitación: una mirada más profunda a las implicaciones del trabajo educativo con el otro y el “...otro son aquellos alumnos y alumnas que desde su modo de estar en el mundo cuestionan la pedagogía, porque hacen tambalear sus principios con su sola presencia en las aulas" (Pérez, 2009, p. 45).

A partir de lo presentado habría que preguntarse por el concepto de la normalidad: ¿quién la inventa?, ¿desde dónde? y ¿para qué? Habría que detenerse en el instante en que el cuerpo humano (y el humano mismo) dice a otro cuerpo que se trata 
"Habría que comprender al otro más allá del aquí y del ahora en el que ha sido señalado como otro, sin desconocer la distancia que nos separa, que históricamente nos ha separado, una distancia comunicativa, cognitiva, cultural, social, económica que no es insalvable si se reconoce que eso otro también está dentro de mí..."

de un ser inhumano. El momento en que cualquier cuerpo es la oposición de nuestro cuerpo. (Cfr. Skliar, 2006a, p. 192). Habría que comprender al otro más allá del aquí y del ahora en el que ha sido señalado como otro, sin desconocer la distancia que nos separa, que históricamente nos ha separado, una distancia comunicativa, cognitiva, cultural, social, económica

\section{Bibliografía}

Álvarez, A. (1995), Y la escuela se hizo necesaria, Bogotá, Magisterio.

Bhaba, H. (1994), The Location of culture, New York, Routledge.

Castro-Gómez, S. (2005), “Ciencias sociales, violencia epistémica y el problema de la invención del otro”, en Lander, E. (comp.), La colonialidad del saber; eurocentrismo y ciencias sociales, Buenos Aires, Clacso, pp. 145-161.

Duschatzky, S. (1999), La escuela como frontera, Buenos Aires, Paidós.

Duschatzky, S. y Skliar, C. (2001), "Los nombres de los otros. Narrando a los otros en la cultura y en la educación”, en Larrosa, J.y Skliar, C. Habitantes de Babel. Políticas y poéticas de la diferencia, Barcelona, Laertes. pp. 185-212.

Dussel, E. (2006), Tesis de política, México, Crefal - Siglo XXI.

Dussel. I. y Gutiérrez, D. (2006), Educar la mirada. Políticas y pedagogías de la imagen, Buenos Aires, Flacso.

Gentili, P. (2000), Códigos para la ciudadanía, Buenos Aires, Santillana.

Guido, S. et al. (2010), Representaciones pedagógicas, políticas públicas y experiencias educativas sobre educación intercultural en la ciudad de Bogotá. Informe de investigación, Bogotá, CIUP, Universidad Pedagógica Nacional [Inédito].

Larrosa, J. y Skliar, C. (2001), Habitantes de Babel. Políticas y poéticas de la diferencia, Barcelona, Alertes.

Larrosa, J. y Skliar, C. (2009), Experiencia y alteridad en Educación. Buenos Aires, Flacso.

Larrosa, J. y Pérez, N. (1998), Imagens do outro, Petrópolis, Editora Vozes.

Lino, N. (2000), "Iguales y diferentes: escuela y diversidad cultural” en Gentili. P. Códigos para la ciudadanía, Buenos Aires, Santillana. que no es insalvable si se reconoce que eso otro también está dentro de mí, que el otro es necesario más allá del conocimiento teórico, de las metodologías, para descifrar otras partes de mi, para completarme. Las distancias se salvan por una relación en la que todos somos seres humanos, en la que acogemos al otro desde el conocimiento de su singularidad.
León, E. (2009), Los rostros del otro, México, Anthropos.

Pérez, N. (2009), “Escuchar al otro dentro de sí” en Larrosa, J. YSkliar, C. Experiencia y alteridad en Educación, Buenos Aires, Flacso.

Pineau, P. (1996), "La escuela en el paisaje moderno. Consideraciones sobre el proceso de escolarización”, [en línea], disponible en: http://www.unlu.edu.ar/ histelea/pdf/ pineau01.pdf, recuperado: 20 demayo de 2010.

Sabido, O. (2009), "El extraño" en León, E. Los rostros del otro, México, Anthropos.

Skliar, C. (2000), “Discursos y prácticas sobre la deficiencia y la normalidad. Las exclusiones del lenguaje, del cuerpo y de la mente" en Gentili. P., Códigos para la ciudadanía, Buenos Aires, Santillana.

Skliar, C. (2004), Y si el otro no estuviera ahí, Buenos Aires, Miño Dávila.

Skliar, C. (2006), "La educación (que es del otro). Notas acerca del desierto argumentativo en Educación" en Educación y pedagogía, Separata, Medellín, Universidad de Antioquia.

Skliar, C. (2006a), "Palabras de la normalidad. Imágenes de la anormalidad" en Dussel. I. y Gutiérrez, D. Educar la mirada. Políticas y pedagogías de la imagen, Buenos Aires, Flacso.

Skliar, C. (2008), “ ¿Qué pretendemos con la idea de diversidad? ¿Qué pretende la idea de diversidad con nosotros?”, [en línea], disponible en: http://www.salesianoslitoral. org.ar/files/sei/2008-encuentro_equipos/4-skliar_carlos_la_idea_de_diversidad.doc, recuperado: 19 de marzo de 2010.

Walkman, G. (2009), "El rostro en la frontera”, en León, E. Los rostros del otro, México, Anthropos. 


\section{Comprensión de los procesos de 10010101010}

\section{educativa de estudiantes sordos desde una}

\section{perspectiva organizacional}

Comprehension of processes of educative inclusion of deaf students from an organizational perspective

\section{Compreensão dos processos de inclusão educacional de alunos surdos desde uma perspectiva organizacional}

\section{Pablo Alejandro Salazar Restrepo Rita Flórez Romero* Clemencia Cuervo Echeverri**}

Magíster en Educación, Universidad Nacional de Colombia. Rector del INEM Francisco de Paula Santander, Bogotá D. C. En el momento de realización del trabajo era rector de la Institución Educativa Distrital Federico García Lorca.

Correo electrónico: pasalazarr@unal.edu.co

** Magister en Lingüística, Universidad Nacional de Colombia. Profesora Asociada, Departamento de Comunicación Humana, Facultad de Medicina, Universidad Nacional de Colombia. Investigadora de los grupos "Cognición y lenguaje en la infancia" y "Oralidad, escritura y otros lenguajes". Coordinadora académica de la Línea de Investigación "Comunicación y educación" de la Maestría en Educación en la misma universidad.

Correo electrónico:rflorezr@unal.edu.co

* Magister en Desórdenes de la Comunicación, Universidad de Illinois, Estados Unidos. Magíster en Lenguaje en la Educación, Universidad de Southampton, Gran Bretaña. Profesora Emérita, Departamento de Comunicación Humana, Facultad de Medicina, Universidad Nacional de Colombia.

Correo electrónico: ccuervoe@cable.net.co

\section{Resumen}

Este artículo parte de la investigación señalada con el mismo nombre en donde se analizan procesos de inclusión de estudiantes sordos del colegio Federico García Lorca desde una perspectiva organizacional. El estudio recurrió a una encuesta que analizó dos variables: estructuras y prácticas organizacionales. Los análisis se contrastaron en grupos focales con la percepción de directivos, docentes, estudiantes, padres de familia y personal administrativo. La inclusión cuenta con un compromiso importante entre miembros de la comunidad educativa, aunque se hace necesario fortalecer el trabajo cooperativo entre los docentes.

\section{Palabras Clave}

Estudiantes sordos, inclusión educativa, perspectiva organizacional, índice de inclusión.

\section{Abstract}

This paper is based on a research which has the same name that analyzes inclusive educative processes for deaf students at Federico Garcia Lorca School from an organizational perspective. This research has recourse to a survey, analyzing two variables: organizational structures and organizational practices. In focal groups the analyses were contrasted with principals', teachers', students', parents' and administrative staff's perceptions. Inclusion counts on an important compromise among members of the educative community, although it is necessary to strength cooperative work among the teachers.

\section{Key Words}

Deaf students, educative inclusion, organizational perspective, index of inclusion.

\section{Resumo}

Este artigo se baseia na pesquisa do mesmo nome, onde se discutem processos de inclusão de estudantes surdos do colégio Federico García Lorca a partir de uma perspectiva organizacional. 0 estudo utilizou uma sondagem que analisou duas variáveis: estruturas e práticas organizacionais. As análises foram contrastadas com as percepções de diretores, professores, alunos, pais e funcionários em grupos focais. A comunidade educacional é muito comprometida com a inclusão, mesmo que é necessário reforçar o trabalho cooperativo entre professores.

\section{Palavras-chave}

Alunos surdos, inclusão educacional, perspectiva organizacional, Índice de inclusão.

Fecha de recepción: 18 de septiembre de 2009 Fecha de aprobación: 15 de diciembre de 2009

Pedagogía y Saberes No.32 Universidad Pedagógica Nacional. Facultad de Educación. 2010, pp. $73-86$ 


\section{Introducción}

$\mathrm{A}$ partir de la década de los años 90 del siglo pasado, se ha establecido la necesidad de atender e integrar poblaciones con "Necesidades educativas especiales" -NEE- en los sistemas educativos de los países; de ahí la preocupación cada vez mayor por incluir este aspecto en las políticas públicas. En tal sentido, se promulgaron legislaciones para atender la problemática y para establecer los parámetros de educación de esta población desde el nivel preescolar hasta el bachillerato; asimismo, se dio impulso a investigaciones y a evaluaciones de impacto de tales procesos. En el caso de Colombia, obedece, además, a la necesidad de garantizar derechos educativos a personas consideradas en situación de discapacidad, vulnerabilidad o desventaja (Sánchez, 2004; Clark, 1999; Ley General de Educación, 1994; Constitución Política de Colombia, 1991). En tal sentido, el trabajo adelantado por el Instituto Nacional Para Sordos -INSORsirvió de punto de partida para la implementación de modelos pedagógicos en el país (INSOR, 2002).

En Bogotá, un estudio acerca del proceso de integración educativa de personas sordas en los colegios de la ciudad (Médicis y Flórez, 2007) arrojó resultados relacionados con la necesidad de profundizar en investigaciones que analizaran y propusieran cambios organizacionales en el ámbito escolar con el fin de optimizar la atención educativa a estudiantes sordos (Clark, 1999).

Los cambios desarrollados en la escuela inclusiva contemplan dos aspectos: por una lado, las estructuras organizacionales y, por otro, las prácticas organizacionales; aspectos que se interrelacionan y dependen en sus procesos, pues buscan el éxito escolar de los alumnos y la satisfacción de las necesidades de todos sus miembros; además, deben constituirse en la base del direccionamiento del proyecto educativo institucional (Sánchez, 2004).

La escuela como organización se convierte en una realidad socialmente construida por sus miembros mediante procesos de interacción social y en relación con los contextos y ambientes donde funciona. En consecuencia, genera estructuras y prácticas informales en el seno de la estructura formalmente reglamentada (Ainscow, 2001).
En tal sentido, la escuela funciona según determinados criterios (Clark y otros, 1999):

a. Administración para la eficiencia: preocupación por prácticas y estructuras organizacionales adecuadas.

b. Administración para la eficacia: logro de los objetivos educativos por medio del adecuado manejo de los recursos.

c. Administración para la relevancia socio-cultural: importancia o pertinencia de los actos y en las prácticas para la vida de los participantes de la comunidad y del sistema educativo.

Asimismo, el paradigma organizacional parte de tres supuestos básicos (Clark y otros, 1999):

1. El ser humano, como ente individual y social políticamente engranado en la sociedad, constituye la razón de ser de la existencia del sistema educativo.

2. La escuela posee estructuras y prácticas diseñadas para preservar la comodidad y la estabilidad del sistema.

3. Las actividades administrativas configuran realidades globales constituidas por dimensiones o planos variados, los cuales se encuentran intrínsecamente relacionados y se benefician o se afectan de manera interdependiente. La escuela, como organización, no se halla ajena a los cambios fundamentales de la sociedad local y global.

La perspectiva o paradigma organizacional en una escuela que implementa un enfoque inclusivo con prioridad por la población con NEE parte de que tales necesidades pueden conformar las prácticas y estructuras organizacionales de las instituciones (Ainscow, 2001; Clark y otros, 1999; Flórez, Moreno, Camacho, Mesa, y Lancheros, 2006). Cuando no se asume la perspectiva organizacional, la flexibilidad curricular queda a criterio del docente y para la institución la atención a las NEE deja de ser una prioridad. Una institución educativa que se asuma como integradora debe concentrarse más en las formas como está organizada para atender a estos estudiantes y poder reorganizarse.

Una escuela inclusiva ha de tener claro que el éxito alcanzado en su labor depende, en gran medida, del enfoque administrativo que asuma. El liderazgo se constituye en una de las características más importante en la escuela y debe ser ejercido como una labor conjunta asumida por diversos miembros de la comunidad que apropien y asuman como suyo el proyecto (Ball, 1989). Una estructura organizacional en las escuelas inclusivas requiere

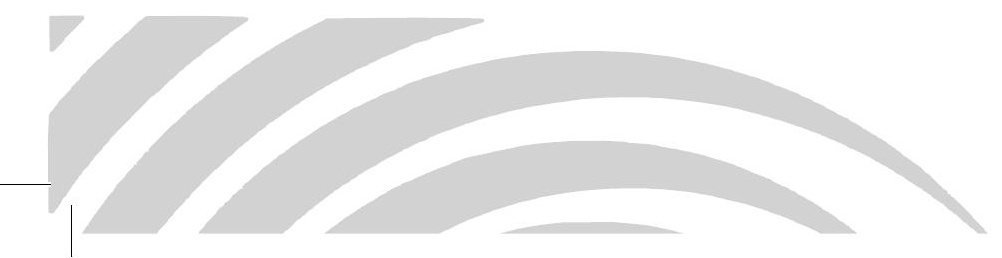


lograr que los grupos de trabajo den el máximo de sus posibilidades en la consecución de las metas trazadas y, de este modo, favorecer la satisfacción y la realización de cada uno de los miembros de la comunidad educativa.

Se torna necesario partir, entonces, de una realidad latente en las comunidades educativas tradicionales: el rechazo, casi automático, frente a la posibilidad de que la escuela de sus hijos o donde se trabaja se halle interesada en orientarse hacia la inclusión de todo tipo de población. En general, los docentes, en principio, y luego los padres se rehúsan a esta transformación. Tanto el trabajo colaborativo como la conformación de equipos juegan un rol importante que permite abrir espacios de discusión, de aclaración de los retos que se enfrentan al asumir un proyecto de tales características (Clarky Cools, 1999). La relación con la comunidad vecina se convierte en un frente importante de trabajo en la estructura organizacional (Clark y Cools, 1999).

Una escuela inclusiva debe tener también prácticas inclusivas para asegurar el aprendizaje de sus estudiantes (Ainscow, 2001). Debe contemplar la flexibilidad, la diversificación de los saberes, los ritmos de aprendizaje, la planeación compartida, la transdisciplinariedad, el trabajo docente por grupos multidisciplinarios, unas formas de evaluación innovadoras, la ruptura de las tradiciones en cuanto a horarios estrictos, tiempos de programación inmodificables, las rutinas de las tareas escolares, la individualización de las enseñanzas y los aprendizajes y los métodos de instrucción diferenciada (Hall, 2002; Liston, 2006; Potter, 2008).

El trabajo pedagógico implica, asimismo, cooperación con los docentes. Una escuela inclusiva requiere, necesariamente, docentes que sientan como propio el proyecto de inclusión, que lo hagan parte de su quehacer cotidiano (Stainback, 2001). Los gobiernos deben centrar sus esfuerzos en mejorar las condiciones laborales de los docentes y en brindarles procesos de capacitación permanente que les permitan acceder a nuevos conocimiento y a cualificar sus prácticas pedagógicas (Ainscow, 2001; Potter, 2008). Ahora bien, la capacitación señalada ha de propender por la sensibilización hacia la inclusión, pues distintos autores (Flórez, Moreno, Camacho, Mesa y Lancheros, 2006; Varma, 2007) coinciden en afirmar que uno de los mayores obstáculos en la inclusión educativa se da en el rechazo de los docentes a trabajar con estudiantes con discapacidad, dado los temores y el desconocimiento de los mecanismos de adaptación curricular y de las evaluaciones.
"Se torna necesario partir, entonces, de una realidad latente en las comunidades educativas tradicionales: el rechazo, casi automático, frente a la posibilidad de que la escuela de sus hijos o donde se trabaja se halle interesada en orientarse hacia la inclusión de todo tipo de población. En general, los docentes, en principio, y luego los padres se rehúsan a esta transformación."

El trabajo de los docentes a través de los proyectos cooperativos se constituye en otra de las estrategias pedagógicas que caracterizan una escuela inclusiva desde la perspectiva organizacional (Ainscow, 2001; Alberta Ministry of Education, 2006; Sánchez, 2004; Stainback, 2001; Varma, 2007). Lo anterior implica, entonces, que los docentes se incluyan en las actividades educativas propias de la escuela (Ainscow, 2001; Arnaiz, 2004; Liston, 2006; Martín, 2006).

Las practicas organizacionales involucran otros dos factores en el proyecto de inclusión: el presupuesto y las adecuaciones físico-ambientales (Sánchez, 2004). El presupuesto varía de acuerdo con las políticas y la normatividad vigente en cada país: en Gran Bretaña y Estados Unidos, por ejemplo, las escuelas que atienden población con NEE reciben un presupuesto especial destinado al equipamiento, la preparación del profesorado y a los demás requerimientos que se tengan (Stainback, 2001). En Colombia no existe una destinación específica para estos fines, aunque los organismos estatales realizan esporádicamente dotaciones de equipo y procesos de formación a los docentes.

Para caracterizar la perspectiva organizacional se adaptó el Index for Inclusion (Booth y Ainscow, 2000), o La guía para la evaluación y mejora de la educación inclusiva. Se cuenta, así, con una herramienta que permite analizar, mediante una serie de indicadores, las estructuras y las prácticas organizacionales características de una institución, incluidas las percepciones de sus actores. A partir de un conjunto de indicadores y preguntas, el centro educativo puede realizar un análisis de su situación presente y de sus posibilidades futuras de cara a una mayor inclusión. 
El documento consta de tres partes. La primera señala brevemente algunos aspectos teóricos que fundamentan el Index; en una segunda, se expone propiamente la organización del Index; y, por último, se explica la metodología de aplicación del instrumento. Asimismo, el documento plantea una perspectiva de derechos respecto de los estudiantes en desventaja educativa que se observa mediante el concepto de "barreras para el aprendizaje y la participación" (Sandoval, 2002).

Cada institución puede seleccionar aquellos indicadores o aspectos que consideren importantes trabajar, pues el instrumento es flexible y se adecúa a las necesidades del contexto de la escuela (Booth y Ainscow, 2000; Durán, 2005; Martín, 2006; Sandoval, 2002). El instrumento consta de aproximadamente 450 ítems dados en forma de indicador o de pregunta; su implementación total demanda un proceso a mediano plazo.

La presente investigación buscó comprender y caracterizar el proceso de atención educativa de la población sorda que se lleva a cabo en la Institución Educativa Distrital Federico García Lorca. La hipótesis inicial del estudio señala que no basta con integrar estudiantes a las aulas regulares en los colegios; es necesario crear estructuras y prácticas organizacionales adecuadas para realizar la inclusión educativa (Clark et al, 1999).

\section{Método, diseño y procedimiento}

La investigación se basó, según se ha venido exponiendo, en el paradigma organizacional escolar para aportar en la comprensión de los procesos de inclusión educativa de estudiantes sordos a partir de una perspectiva descriptiva adaptada al hecho educativo. Consistió en un estudio descriptivo con elementos de acción participante y de confrontación etnográfica de los resultados obtenidos con los instrumentos.

En el estudio se caracterizaron las estructuras y prácticas de la IED Federico García Lorca a través de diferentes actores participantes de dicha organización. Para lograr el objetivo, se desarrollaron once pasos:

- Conformación del grupo interdisciplinario mediante convocatoria a aquellas personas interesadas y que tuvieran conocimientos en el tema del estudio (dos sesiones de trabajo de dos horas cada una).

- Socialización del estudio y acercamiento a la propuesta por medio de presentación y sensibilización de la investigación al grupo interdisciplinario (una sesión de cuatro horas).
“La investigación se basó, en el paradigma organizacional escolar para aportar en la comprensión de los procesos de inclusión educativa de estudiantes sordos a partir de una perspectiva descriptiva adaptada al hecho educativo. Consistió en un estudio descriptivo con elementos de acción participante y de confrontación etnográfica de los resultados obtenidos con los instrumentos."

- Acercamiento al documento Index For Inclusion mediante su presentación y distribución (tres horas).

- Análisis del documento y selección de indicadores pertinentes para la investigación (cinco horas).

- Discusión de indicadores y construcción de una primera versión. El borrador se envió a los miembros del grupo interdisciplinario quienes formularon las observaciones pertinentes ( 20 horas).

- Aprobación de la encuesta para pilotaje, una vez recibidas las observaciones (diez horas).

- Pilotaje de la encuesta por medio de su aplicación a un pequeño grupo (tres horas).

- Adecuación de la encuesta de acuerdo con el pilotaje (20 horas).

- Aplicación de la encuesta en grupos pequeños por estamentos, a docentes y directivos, estudiantes sordos, estudiantes oyentes, padres de familia y personal administrativo y de servicios. Cada uno de ellos tomó entre una hora y hora y media para su diligenciamiento ${ }^{1}$.

- Análisis de datos mediante el programa estadístico "Statiscal Programe for Social Sciences" (SPSS). El análisis factorial tomó como base dos categorías: las estructuras y las prácticas organizacionales. Las variables que aportaron un mayor peso en estos factores determinaron las características que se debían tener en cuenta

1 Durante el pilotaje de la encuesta al estudiante sordo se observó la gran dificultad que tuvo para la comprensión de los textos, por esto se hizo necesario la elaboración de un video de la encuesta en el cual a través de la interpretación de los textos en LSC se logró por un lado disminuir el tiempo empleado para la solución de la encuesta de 2 horas a 45 minutos y por otro lado la comprensión de la encuesta en el marco general de los procesos de inclusión. 
al final del estudio. Una vez obtenida la información estadística, se procedió a analizar e interpretar los hallazgos a la luz de los fundamentos de la perspectiva organizacional.

- Validación de los resultados con un grupo focal integrado por expertos en el tema de inclusión al interior del colegio. El grupo contó con la participación de un directivo coordinador, dos docentes de apoyo, un docente de educación media con experiencia en temas de inclusión, un intérprete de lengua de señas, un estudiante sordo de educación media y un estudiante oyente. El trabajo del grupo focal se centró en la implementación de un plan de mejoramiento institucional.

\section{Participantes}

Para el estudio se conformaron dos conjuntos de participantes. Un grupo interdisciplinario integrado por uno de los investigadores principales, dos docentes de educación básica que atienden estudiantes sordos en aula multigrados, una docente que trabaja con estudiantes sordos incluidos en aula de educación media, una fonoaudióloga y dos intérpretes de lengua de señas. Para la selección de la muestra se utilizó la técnica de muestreo deliberado o por conveniencia (Babbie, 2001). La tabla 1 relaciona los participantes:

Tabla 1. Muestra de participantes en el estudio

\begin{tabular}{|l|c|}
\hline Estamento & Encuestados \\
\hline Directivos-coordinadores & 2 \\
\hline Docentes de aula sordos & 1 \\
\hline $\begin{array}{l}\text { Docente profesional de apoyo } \\
\text { (Fonoaudióloga) }\end{array}$ & 1 \\
\hline $\begin{array}{l}\text { Docente de castellano } \\
\text { como segunda lengua }\end{array}$ & 4 \\
\hline $\begin{array}{l}\text { Docentes de primaria } \\
\text { sin contacto con sordos }\end{array}$ & 5 \\
\hline $\begin{array}{l}\text { Docentes de Bachillerato } \\
\text { que trabajan con estudiantes sordos }\end{array}$ & 4 \\
\hline $\begin{array}{l}\text { Docentes de Bachillerato } \\
\text { sin contacto con sordos }\end{array}$ & 4 \\
\hline $\begin{array}{l}\text { Padres de familia de estudiantes } \\
\text { sordos bachillerato }\end{array}$ & 6 \\
\hline $\begin{array}{l}\text { Padres de familia de estudiantes } \\
\text { sordos de primaria }\end{array}$ & 5 \\
\hline Estudiantes sordos de bachillerato & 2 \\
\hline $\begin{array}{l}\text { Estudiantes Bachillerato } \\
\text { comparten curso con sordos }\end{array}$ & 4 \\
\hline
\end{tabular}

\begin{tabular}{|l|c|}
\hline $\begin{array}{l}\text { Estudiantes Bachillerato } \\
\text { con algún contacto con sordos }\end{array}$ & 5 \\
\hline Estudiantes sordos 5o primaria & 5 \\
\hline $\begin{array}{l}\text { Interpretes de lengua de señas } \\
\text { colombiana }\end{array}$ & 2 \\
\hline Modelos lingüísticos & 2 \\
\hline $\begin{array}{l}\text { Personal administrativo, } \\
\text { secretaria y bibliotecario }\end{array}$ & 3 \\
\hline $\begin{array}{l}\text { Personal de apoyo, aseadoras y } \\
\text { celadores }\end{array}$ & 62 \\
\hline Total de Encuestas & 3 \\
\hline
\end{tabular}

El segundo conjunto de participantes se conformó con 34 padres y estudiantes del colegio: diez padres de familia de estudiantes sordos, catorce estudiantes sordos y diez estudiantes oyentes. También respondieron la encuesta 24 docentes y directivos docentes y seis funcionarios administrativos de la IED Federico García Lorca de Bogotá, D. C.

\section{Instrumentos}

El grupo interdisciplinario diseñó una encuesta a partir de la selección y la adaptación de algunos indicadores del ya señalado Index for Inclusion.

La adaptación del instrumento llevó a la construcción de tres encuestas destinadas a los diferentes grupos de miembros de la comunidad educativa: directivos y docentes, estudiantes y padres de familia y personal administrativo y de servicios generales. La encuesta 1, "Directivos y docentes", se estructuró de la siguiente forma:

Tabla 2. Estructura de los instrumentos aplicados

\begin{tabular}{|c|c|c|}
\hline Dimensión & Secciones & $\begin{array}{c}\text { No de } \\
\text { Preguntas }\end{array}$ \\
\hline \multirow{8}{*}{$\begin{array}{l}\text { Estructura } \\
\text { Organizacional }\end{array}$} & Gobierno escolar & 4 \\
\hline & $\begin{array}{l}\text { Inclusión y perma- } \\
\text { nencia de docentes }\end{array}$ & 6 \\
\hline & $\begin{array}{l}\text { Ingreso de } \\
\text { estudiantes }\end{array}$ & 8 \\
\hline & $\begin{array}{l}\text { Apoyo a la } \\
\text { diversidad }\end{array}$ & 3 \\
\hline & $\begin{array}{l}\text { Apoyos } \\
\text { profesionales }\end{array}$ & 3 \\
\hline & Formación docente & 5 \\
\hline & $\begin{array}{l}\text { Estudiantes } \\
\text { con N.E.E. }\end{array}$ & 10 \\
\hline & Intimidación & 3 \\
\hline
\end{tabular}




\begin{tabular}{|c|c|c|}
\hline \multirow{6}{*}{$\begin{array}{l}\text { Prácticas } \\
\text { Organizacionales }\end{array}$} & $\begin{array}{l}\text { Procesos de } \\
\text { aprendizaje }\end{array}$ & 12 \\
\hline & $\begin{array}{l}\text { Procesos } \\
\text { convivenciales }\end{array}$ & 3 \\
\hline & $\begin{array}{l}\text { Procesos de } \\
\text { enseñanza }\end{array}$ & 8 \\
\hline & $\begin{array}{l}\text { Actividades } \\
\text { extraescolares }\end{array}$ & 3 \\
\hline & $\begin{array}{l}\text { Recursos } \\
\text { disponibles }\end{array}$ & 4 \\
\hline & Total Preguntas & 72 \\
\hline Dimensión & Secciones & $\begin{array}{c}\text { No de } \\
\text { Preguntas }\end{array}$ \\
\hline \multirow{5}{*}{$\begin{array}{l}\text { Estructura } \\
\text { Organizacional }\end{array}$} & Gobierno escolar & 4 \\
\hline & $\begin{array}{l}\text { Políticas de } \\
\text { inducción }\end{array}$ & 4 \\
\hline & $\begin{array}{l}\text { Apoyo a la } \\
\text { diversidad }\end{array}$ & 3 \\
\hline & $\begin{array}{l}\text { Apoyos } \\
\text { profesionales }\end{array}$ & 3 \\
\hline & $\begin{array}{l}\text { Estudiantes } \\
\text { con N.E.E. }\end{array}$ & 5 \\
\hline \multirow{6}{*}{$\begin{array}{l}\text { Prácticas } \\
\text { Organizacionales }\end{array}$} & $\begin{array}{l}\text { Procesos de } \\
\text { aprendizaje }\end{array}$ & 7 \\
\hline & $\begin{array}{l}\text { Procesos } \\
\text { convivenciales }\end{array}$ & 2 \\
\hline & $\begin{array}{l}\text { Procesos de } \\
\text { enseñanza }\end{array}$ & 4 \\
\hline & $\begin{array}{l}\text { Actividades } \\
\text { extraescolares }\end{array}$ & 2 \\
\hline & $\begin{array}{l}\text { Recursos } \\
\text { disponibles }\end{array}$ & 2 \\
\hline & Total Preguntas & 36 \\
\hline
\end{tabular}

"La investigación se basó, según se ha venido exponiendo, en el paradigma organizacional escolar para aportar en la comprensión de los procesos de inclusión educativa de estudiantes sordos a partir de una perspectiva descriptiva adaptada al hecho educativo. Consistió en un estudio descriptivo con elementos de acción participante y de confrontación etnográfica..."
La encuesta 3, "Personal administrativo y de servicios generales", presentó la siguiente estructura:

\begin{tabular}{|l|c|}
\hline Dimensión & No de Preguntas \\
\hline Estructura Organizacional & 6 \\
\hline Practicas Organizacionales & 3 \\
\hline Total Preguntas & 9 \\
\hline
\end{tabular}

\section{Resultados}

Los resultados se organizaron según los actores que respondieron encuestas, actores que se categorizaron en atención del Index for Inclusion: padres y estudiantes, docentes y directivos docentes y funcionarios administrativos. De cada grupo se describen, a continuación, las respuestas dadas en relación con la estructura y las prácticas organizacionales.

\section{Encuestas aplicadas a padres y estudiantes}

\section{Dimensión estructura organizacional}

\section{Gobierno escolar.}

- El 85 \% de encuestados conoce el proyecto de inclusión con o sin participación en él.

- Uno de cada tres padres, en promedio, participa solo.

- Los padres mencionan diferentes razones para su participación, todas referidas al ejercicio de liderazgo.

- De los catorce estudiantes sordos, cinco afirman participar en la construcción de las políticas pero no dicen cómo o de qué manera.

- Los estudiantes oyentes mencionan razones con un componente inclusivo como "Nos han dado posibilidad de compartir, de estar en relación con ellos".

- El 60\% de los encuestados responde que sí se han creado espacios de participación de los estudiantes sordos. Los que integran el Consejo Directivo se pronuncian favorablemente en el 67,6\%, y en los integrantes del Consejo de Padres y la Asociación de Padres el 58,8\%.

- El 50\% de los encuestados dice que se ven representados en el gobierno escolar; sólo siete manifiestan la razón del porqué de su respuesta. 


\section{Políticas de inducción}

De acuerdo con los resultados obtenidos en la encuesta, uno de los principales puntos que deben ser tenidos en cuenta en la elaboración del plan de mejoramiento consiste en implementar más políticas de inducción, pues los resultados demostraron una carencia al respecto. La figura 2 consolida los resultados de los ítems cinco a siete.

Figura 1. Políticas de inducción (ítems 5 a 7)

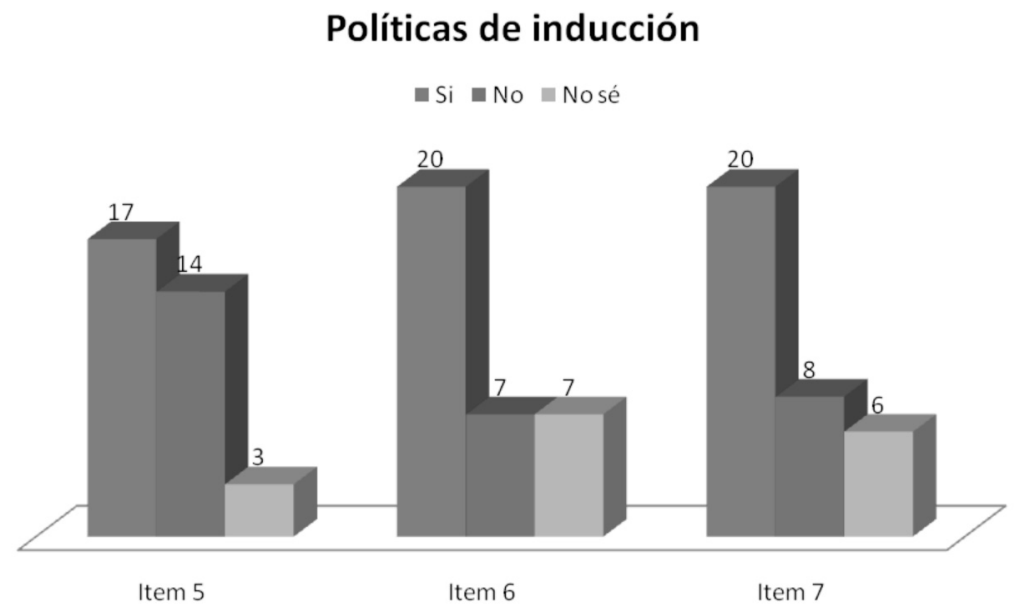

Figura 1. Políticas de inducción (ítems 5 a 7)

5. ¿Usted recibió algún tipo de inducción antes de ingresar al colegio?

6. ¿Se motiva la participación de las familias de estudiantes sordos en las actividades del colegio?

7. ¿Se han establecido programas para facilitar la transición entre el preescolar y primaria y primaria y secundaria para los estudiantes sordos?

Para la mayoría de encuestados, los folletos, seguidos de la página web, se constituyeron en la principal fuente de disponibilidad de información para conocer los fundamentos del colegio.

\section{Apoyo a la diversidad}

Se percibe una mayor consistencia en las respuestas de los padres de estudiantes sordos de secundaria: todos contestan que sí se dan a conocer las leyes y que se incluye a los estudiantes en clases y en otras actividades escolares, así como a ellos mismos. Reconocen las electivas, los juegos y los deportes como las actividades donde son incluidos los estudiantes sordos; por último, se tienen en cuenta las clases.

\section{Apoyos profesionales}

- El 82\% de padres y estudiantes conoce la existencia del equipo de profesionales de apoyo; el $74 \%$ de los encuestados considera que estos apoyos han facilitado la integración de los estudiantes sordos.
- Un total de seis estudiantes sordos reconocen la existencia del equipo, pero sólo la mitad de ellos considera que han facilitado la integración. La restante población encuestada manifiesta un resultado consistente y positivo hacia el aporte del equipo de apoyo profesional.

- El $65 \%$ de padres y estudiantes no conoce la gestión operativa del equipo profesional de apoyo.

\section{Estudiantes con necesidades educativas especiales}

- La percepción hacia los estudiantes sordos es positiva en padres y estudiantes.

- Sólo un estudiante sordo y un estudiante oyente de otro curso respondieron que en el colegio los estudiantes sordos son considerados como alumnos especiales con quienes se ha de tener preferencia y "cierta lástima".

- El 53\% de encuestados responde no, no se o no responde en relación con la consideración de que los estudiantes sordos son alumnos con diferentes intereses, conocimientos, y habilidades. 


\section{Dimensión prácticas organizacionales}

\section{Procesos de aprendizaje}

- Los procesos de aprendizaje se perciben de manera diferente por padres y estudiantes; para los padres los objetivos pedagógicos de las actividades son claros y existen oportunidades para realizar tareas de diferentes maneras.

- En el 85 \% de padres y estudiantes concuerdan los aspectos referidos a la participación en igualdad de condiciones de los estudiantes sordos en las clases.

- El $71 \%$ de encuestados afirma que los estudiantes sordos se tienen en cuenta para organizar las actividades diferentes de las clases.

- Menos del 50\% de los encuestados señala que se emplean estrategias diferentes donde los estudiantes sordos pueden demostrar sus aprendizajes.

\section{Procesos de convivencia}

- Los estudiantes sordos se perciben como excluidos, pero la mayoría de padres considera que sí se les involucra.

- Solo el 50 \% valora el "Manual de convivencia" como herramienta útil para tratar los conflictos de los estudiantes sordos.

- El $76 \%$ de estudiantes dice entender el Manual.

\section{Procesos de enseñanza}

- Menos del 50\% considera que se tienen en cuenta en la planeación o en la evaluación del año escolar.

- El 91 \% reconoce la importancia de aprender la lengua de señas.

- Se percibe mayor claridad en la función de los intérpretes que en la de las fonoaudiólogas en diferentes aspectos del proceso de inclusión.

\section{Actividades extraescolares}

Padres y estudiantes consideran que los estudiantes sordos cuentan con oportunidades de participación en actividades deportivas (82,4\%), académicas $(76,5 \%)$ y culturales $(76,5 \%)$.

El $82 \%$ coincide en que las asignaturas electivas permiten el desarrollo de las capacidades de los estudiantes sordos.

\section{Recursos disponibles}

- El 50\% considera que los recursos del colegio posibilitan la inclusión de los estudiantes sordos.

- Una cuarta parte responde que se ha sentido apoyada y que ha recibido orientación cuando lo ha requerido.

\section{Encuestas aplicadas a docentes y a directivos docentes}

Se aplicó un total de 24 encuestas al personal docente de la IED Federico García Lorca. La muestra se considera representativa porque incluye la totalidad de los funcionarios que se relacionan de manera directa con los estudiantes sordos (coordinadores, docentes que les dan clase, modelos lingüísticos, intérpretes y fonoaudióloga). Asimismo, responden el 33\% de los docentes de básica y el $26 \%$ de educción media que no comparten clases con esta población.

\section{Dimensión estructura organizacional}

- El $54 \%$ de docentes considera que existe un plan de inclusión de estudiantes sordos de corto, mediano y largo plazo.

- El 88\% de docentes cree que los estudiantes sordos son tenidos en cuenta para la conformación del gobierno escolar.

- El $67 \%$ de los docentes percibe que existe participación de los padres de estudiantes sordos para la conformación del gobierno escolar.

- El $75 \%$ afirma que los estudiantes sordos participan en este gobierno.

- Para el caso del conocimiento de actividades institucionales, las respuestas plantean un componente inclusivo pues manifiestan una participación "igual que los oyentes no hay diferencia"; algunas respuestas señalan la participación de los intérpretes como elemento dinamizador de la comunicación con los estudiantes sordos.

- El $75 \%$ indica que las decisiones respecto del proceso de inclusión se realizan de manera consultada.

- El 71 \% se siente representado en el gobierno escolar. El restante porcentaje refiere razones de tipo administrativo para no sentirse representado en el gobierno.

- Los docentes que se sienten representados manifiestan dos razones: elección legítima de representantes y facilidad en procesos de comunicación eintercambios de información con el gobierno escolar. 


\section{Inclusión y permanencia de docentes}

El $83 \%$ de los docentes califica de manera positiva la estabilidad del equipo de inclusión; el 88 \%, positivamente la continuidad de estas acciones. A pesar de lo anterior, los docentes evidencian limitaciones de tipo administrativo en la realización de este proyecto; sólo dos terceras partes perciben la inclusión como una oportunidad de desarrollo profesional.

\section{Ingreso de estudiantes}

- Un tercio de los encuestados considera que existe un programa de inclusión de estudiantes sordos.

- Los medios de información general acerca del proyecto se limitan a folletos.

- Nueve de los 24 docentes menciona otras opciones; se destacan los servicios religiosos y el trabajo en red.

- El 75\% considera que el colegio cuenta con programas para facilitar la transición de los estudiantes sordos en los diversos niveles educativos.

- El 58\% da respuesta de no y no sé respecto de un incremento en el número de estudiantes sordos en el colegio.

- El 67\% responde no o no sé frente a la existencia de un sistema de información en la localidad que permita el acceso de estudiantes sordos al colegio.

\section{Apoyo a la diversidad}

Existe consenso entre los docentes y los directivos docentes en relación con su preocupación declarada por conocer las leyes en materia de inclusión para las personas en situación de discapacidad y sus efectos en el trabajo pedagógico.

- Existe un acuerdo en torno a tres elementos que definen la organización de los cursos: criterio del docente del aula, condición de discapacidad y rendimiento.

- Los factores de edad y género no se perciben como influyentes; en especial el del género (figura 2).

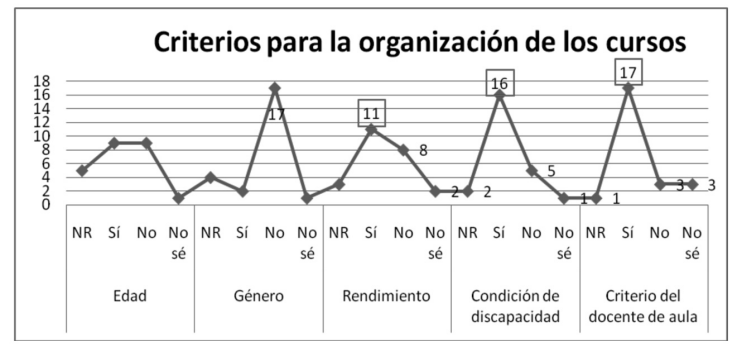

Figura 2. Criterios para la organización de los cursos en el Colegio

- Los encuestados señalan que los estudiantes son incluidos tanto en las clases como en otras actividades.

- Los docentes consideran que los estudiantes sordos participan en actividades como electivas, actividades deportivas, actividades institucionales y culturales (cuatro casos en cada una), las salidas pedagógicas (tres casos), actividades lúdicas (dos casos) y el descanso (un caso).

\section{Apoyos profesionales}

- Los docentes consideran que los apoyos se dirigen hacia la atención y la integración de los estudiantes sordos.

- El 42 \% de docentes considera que los apoyos se concentran en grupos pequeños.

\section{Formación docente}

- Los docentes encuestados manifiestan contar con el tiempo para observar, analizar y reflexionar acerca de las clases; en menor medida, manifiestan la existencia de espacios destinados al trabajo grupal.

- La totalidad de los docentes considera que cuentan con oportunidades de acceso a herramientas tecnológicas para usar en clases; se corrobora con el uso frecuente de apoyos por los docentes de aula de sordos, los intérpretes y los modelos lingüísticos.

- Dos terceras partes de los docentes considera que se emplea el trabajo en grupo como estrategia de capacitación docente.

- La capacitación recibida se centra en la atención a población con limitaciones auditivas; un docente manifiesta haber recibido capacitación en autismo y ceguera. 
- El $58 \%$ de los docentes muestra no haber tenido formación en lengua de señas como medio para mejorar las prácticas pedagógicas.

\section{Estudiantes con necesidades educativas especiales}

- Los docentes y directivos docentes manifiestan una percepción positiva y favorable hacia los estudiantes sordos.

- Dos personas (la profesional de apoyo y un intérprete de lengua de señas) responden que en la institución los estudiantes sordos se consideran como alumnos especiales a quienes "hay que tenerles preferencia y cierta lástima".

- Los docentes de secundaria que imparten clase a estudiantes sordos poco consideran la participación de los estudiantes sordos como una ocasión para mejorar las oportunidades de aprendizaje de los demás estudiantes.

- La totalidad de los docentes considera que el apoyo a los estudiantes con NEE es un derecho.

- El 16,7\% de los docentes considera que el apoyo a los estudiantes con NEE constituye un derecho $\mathrm{y}$ una forma de asistencialismo.

- Un tercio de los docentes creen que los estudiantes sordos y sus padres no son conscientes del apoyo que la institución les da.

- Un tercio de los docentes considera que en la institución se está trabajando por incrementar el aprendizaje y reducir la exclusión.

- Los docentes refieren que, junto con el personal de apoyo, se da atención a los estudiantes sordos en el salón de clases.

\section{Intimidación}

- Dos terceras partes de los docentes consideran que no se manifiestan comentarios discriminatorios acerca de los estudiantes sordos; el 33\% restante, afirma que los comentarios discriminatorios provienen de los mismos docentes.

- El $83 \%$ de los docentes considera que los estudiantes saben a quién acudir en caso de amenazas o intimidación por parte de los compañeros.

- Una cuarta parte de los docentes estima que existe indiferencia y cierto tipo de rechazo hacia los estudiantes sordos en descansos y salidas.

\section{Dimensión prácticas organizacionales}

\section{Procesos de aprendizaje}

- El 79,2 \% de los docentes indica que se tiene en cuenta la diversidad y las experiencias de los estudiantes en la adaptación pedagógica y curricular de las clases.

- El 58,3 \% afirma que se deben identificar y utilizar los intereses de los estudiantes para construir el currículo.

- Los docentes afirman que se efectúan adaptaciones tanto en la presentación de trabajos extraclase (tareas) como en las expectativas del rendimiento escolar de los estudiantes sordos.

- Con miras a su inclusión en las modificaciones y actualizaciones curriculares correspondientes, se considera necesario fortalecer el liderazgo en materia de evaluación para los aprendizajes de los estudiantes y en la sistematización de los hallazgos de estas prácticas.

\section{Procesos de convivencia}

- Los docentes considera que el Manual de Convivencia se torna útil en el momento para abordar los conflictos, $62,5 \%$; para entenderlos, $54,2 \%$; y solucionarlos, 58,3 \%.

- El $50 \%$ de los docentes considera que existen procedimientos claros para solucionar conflictos entre los estudiantes sordos, sus familias y los docentes.

- Dos terceras partes de los encuestados -en especial los coordinadores, los docentes del aula multigrado, los docentes de primaria que no les dan clases a los estudiantes sordos y los modelos lingüísticos- responde que a los estudiantes sordos se les involucra.

\section{Procesos de enseñanza}

- El $50 \%$ de los docentes y directivos docentes no han dispuesto tiempos para la coordinación de docentes que trabajan en el proceso de inclusión. Aducen que no cuentan con los tiempos necesarios para ello en su jornada.

- El $58 \%$ no cree posible realizar una planeación conjunta con la participación de todo el equipo del proyecto de inclusión. 
- Al triangular la información obtenida en las encuestas aplicadas a padres y estudiantes sordos, se ratifica la insuficiencia en tiempos y espacios comunes destinados para la planeación y la coordinación del proyecto.

- El 83,3 \% de los encuestados sostiene que las funciones se hallan suficientemente definidas.

\section{Actividades extraescolares}

Los docentes encuestados perciben un alto nivel de participación de los estudiantes sordos tanto en las actividades académicas (100\%) como en las deportivas y en las asignaturas electivas $(91.7 \%$ en cada una) y, en menor porcentaje, en las actividades extraescolares (no llega al 50\%).

Al triangular la información reportada por el estamento docente con la referida por los padres y estudiantes se corrobora una alta percepción en la comunidad educativa acerca de los niveles de participación en las actividades académicas y deportivas y en la concepción en torno a las asignaturas electivas como potenciadoras para el desarrollo académico de los estudiantes sordos.

\section{Recursos disponibles}

- El $83 \%$ de los docentes valora de forma positiva la participación de familiares en el proceso; y el $30 \%$, la participación de la comunidad.

\section{Encuesta a funcionarios administrativos}

- El $70 \%$ de los funcionarios administrativos no conoce del proyecto de inclusión.

- Ninguno sabe si el Colegio se preocupa por conocer y divulgar las leyes que rigen los procesos de inclusión para las personas en situación de discapacidad.

- Manifiestan no haber recibido capacitación, a pesar de que requieren la interacción continua con estudiantes sordos y con sus familias.

- Todos los funcionarios administrativos encuestados responden: "que no son considerados como alumnos especiales a los cuales hay que tenerles preferencia y cierta lástima".

- Dos de los encuestados manifiestan haber percibido comentarios discriminatorios hacia los estudiantes sordos de parte de los docentes.
- Cinco de los seis encuestados (83\%) manifiestan que "prestan apoyo de forma equitativa independiente de su condición."

La aplicación de este índice permitió identificar que, a pesar de la existencia de unas políticas educativas que promulgan la garantía del acceso y la permanencia de las poblaciones con NEE, el sistema educativo del colegio integra niños y jóvenes con estas condiciones en sus aulas, pero no por ello garantiza su inclusión educativa. Esto se debe a la existencia de algunos vacíos en las estructuras y las prácticas organizacionales para garantizar la equiparación de oportunidades a los estudiantes sordos (Booth y Ainscow, 2000).

\section{Discusión y conclusiones}

El desarrollo de la investigación, de manera cooperativa con el equipo coordinador del Proyecto de Inclusión en el Colegio Federico García Lorca, permitió la participación de la comunidad educativa, el intercambio y la validación de conocimientos y la identificación desde la perspectiva organizacional de las principales fortalezas y de los aspectos que requieren intervención. De esta manera se vuelve posible adaptar las prácticas y estructuras organizacionales de tal modo que permitan la implementación y la materialización adecuada del Proyecto de Inclusión Educativa en la institución. Se observa una consolidación del proyecto de inclusión. Estos proyectos, surgidos inicialmente como compromiso ético de algunos interesados, pueden permear una comunidad educativa e involucrar a todos en un trabajo colaborativo (Potter, 2008).

Respecto de la dimensión de estructura organizacional, la institución, en el lapso de algunos años, ha logrado sufrir unas transformaciones en su proyecto educativo que se pueden observar como positivas. Estas fortalezas se resaltan en distintos aspectos:

El proyecto de inclusión de estudiantes sordos es consultado. Esto difiere de lo reportado en algunas investigaciones (Ainscow, 2001; Varma, 2007) donde se manifiesta que este tipo de decisiones se toman de manera vertical e inconsulta. De acuerdo con algunos autores, las personas con limitaciones deben atenderse necesariamente en el ámbito educativo porque se trata de un derecho fundamental (Pérez y Uprimny, 2007); sin embargo, cuando se ha consolidado un proyecto como iniciativa colectiva, dicho proyecto tiende a tener mayor sostenibilidad en el futuro (Clark, 1999). Este hecho se fortalece porque la percepción predominante en los docentes 
su lengua de señas como en el dominio del español (INSOR, PEBBI, Portilla, Ramírez y Flórez, 2008).

En relación con el último aspecto, resulta relevante indicar que la presente investigación permite vislumbrar varios caminos hacia donde debe enrumbarse la orientación de la IED Federico García Lorca para lograr mayores niveles de eficiencia en el desarrollo del proyecto de inclusión educativa de estudiantes sordos y con necesidades educativas especiales:

- Continuar en la realización de actividades de socialización entre la población sorda y oyente con el fin de crear un ambiente propicio para el desarrollo de procesos comunicativos.

- Generar desequilibrios que obligan a la adaptación, regulación, implementación o adecuación de las prácticas y estructuras organizacionales.

- Priorizar el desarrollo de competencias comunicativas en niños y jóvenes sordos que ingresan a la institución para incluirse con la población oyente.

- No dejar el proyecto de inclusión como responsabilidad exclusiva de un solo (estudiante, maestro, directivo docente o padres de familia).
- Mantener la movilización de la comunidad educativa para contrarrestar la tendencia de las organizaciones a preservar la estaticidad en sus estructuras y prácticas.

- Profundizar más en el estudio y en la investigación de las dificultades de aprendizaje que aparentemente tienen los estudiantes sordos en comparación con los oyentes con el fin de generar acciones de inclusión más acordes con las particularidades de las personas.

- Fortalecer el trabajo cooperativo desarrollado en el grupo de docentes en torno a los procesos con los estudiantes sordos y a los métodos y las didácticas más efectivas en su educación.

- Desarrollar futuras investigaciones en el campo de los procesos comunicativos que se llevan a cabo en el marco de la inclusión educativa, de las estrategias de trabajo colaborativo de docentes y estudiantes, del papel de los padres de familia como mediadores del proceso educativo y del análisis y autoevaluación que deben llevar a cabo las instituciones educativas en el grado de inclusión o exclusión en sus prácticas pedagógicas.

\section{Bibliografía}

Ainscow, M. (2001), Desarrollo de escuelas inclusivas, Madrid, Narcea.

Alberta Ministry of Education (2006), Essential components of educational programming for students who are blind or visually impaired. Edmonton: Alberta Ministry of Education.

Arnaiz, P. (2004), “La educación inclusiva: dilemasy desafíos” en Educación, desarrollo y diversidad, núm.7, 22-40.

Babbie, E. (2001), Fundamentos de la investigación social, Madrid, Cengage Learning.

Ball, S. J. (1989), La micropolítica de la escuela. Hacia una mirada de la organización escolar. Barcelona, Paidós.

Booth, T., y Ainscow, M. (2000), Index for Inclusion, Bristol, Centre for Studies on Inclusive Education.

Clark, K. et al. (1999), "Theories of inclusion, theories of schools: Deconstructing and Reconstructing the 'Inclusive Schools', en British Educational Research Journal, 25(2), pp.157-177.
Durán, D. et al. (2005), “Primeras experiencias de uso de la guía para la evaluación y la mejora de la educación inclusiva (Index for Inclusion) en el estado Español", en Revista electrónica iberoamericana sobre calidad, eficacia y cambio en educación, 3(1), pp. 464-467.

Elliot, J. (1994), La investigación -acción en educación, Madrid, Morata.

Flórez, R. et al. (2006), “Una aproximación conceptual al proceso de integración escolar" en Moreno, M., y Flórez, R. (2006). (comps.) Integración escolar: aprender desde la diferencia, Bogotá: Secretaría de Educación Distrital, Universidad Nacional de Colombia, Red Distrital Aprender desde la diferencia, Red de Maestros y Maestras Integradores REDMAIN.

Flórez, R., y Moreno, M. (2007), Lectura y comunicación en el aula: un ecosistema. Memorias del VII Congreso nacional de Lectura y IV Coloquio Colombo-Francés de Bibliotecas, Bogotá, Fundalectura.

Hall, T. (2002), Differentiated Instruction, Washington, National Center on Accessing the general curriculum. 